\title{
A lesson in communication
}

\author{
Willingness to ask questions and having frank conversations with your collaborators can lead to many \\ opportunities in translational research, reflects James E. Dahlman.
}

l was a sophomore at Wright State University when I heard a talk that changed my scientific life. It was 2007 and I was sitting in an overcrowded lecture hall at the Materials Science and Technology meeting in Detroit. I was there to give an oral presentation on metallic glasses, which I was studying at the Air Force Research Labs. But I distinctly remember Subra Suresh, then the Dean of School of Engineering at the Massachusetts Institute of Technology (MIT), describing how red blood cells stiffened as they were infected with malaria. The cells could no longer contort their way through tiny capillaries that permeate nearly all of our organs, and this poor perfusion prolonged the disease. As he finished his presentation, I realized that techniques developed in engineering departments would be rapidly incorporated into biology, genomics and medicine. This idea captivated me so much that I applied to MIT for graduate studies and began working at the intersection of nanotechnology and medicine in Robert Langer's laboratory.

We were interested in endothelial cells, which are the cells that line blood vessels. Because these cells are everywhere in the body, their dysfunction contributes to many illnesses including stroke, heart disease, emphysema and cancer. On page 648 of this issue, we report nanoparticles that preferentially deliver small interfering RNA (siRNA) molecules into endothelial cells to suppress the expression of specific genes in these cells. Once we identified and optimized these nanoparticles, we shared them with biologists and clinicians who used them to study the role of endothelial cells in diseases. I learned a lot about science and research during the course of this project but most importantly, I realized that as a materials scientist and medical engineer, communicating effectively with biologists and clinicians can lead to many opportunities in translational research.

Exchanging ideas with scientists in different fields can be challenging because common terms and techniques in one field can be completely foreign to another. For example, I described our nanoparticles to biologists as 'nano-gobstoppers'. Because most have eaten this common candy that consists of different layers of colours and flavours, it was easy for them to visualize the

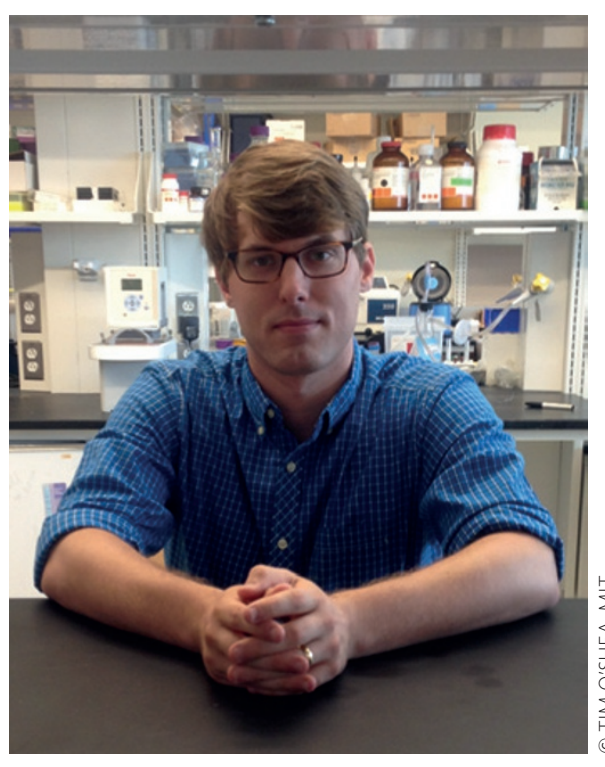

batches. We then studied which cell types the particle could, and could not, deliver drugs to. Once we had a repeatable, consistent and well-characterized particle, we approached biologists and clinicians who could use the technology for their own research. I believe this strategy was critical to our success. Based on all the data generated in our lab, we were confident that the nanoparticles delivered siRNA to endothelial cells in vivo very efficiently. For this reason, we focused on approaching labs that studied endothelial cell function.

Because my collaborators were patient and thoughtful experts in their field, I was able to learn several new concepts and techniques from them. On several occasions, we openly debated and critiqued different experimental ideas and techniques. Coupled with my willingness to ask questions (even when they sounded naive), we were able to significantly improve the quality of our research. Our trust and respect for each other allowed such frank alternating layers of lipid and siRNA in our nanoparticles. Likewise, I first learned about flow cytometry from Aude Thiriot, who is a world-class immunologist and teacher. Aude was surprised that drug-delivery scientists did not use this technique more often, because it could be used to measure the expression of protein from many different types of cell taken from the same tissues. It took me a while to learn how to prepare cells for analysis, but once I did, I realized flow cytometry was a perfect tool for studying which cell types your drug affects. These interactions taught me to answer three questions before embarking on an experiment with my collaborators: what is the assay meant to show? Why is the chosen assay more appropriate than other similar ones? And, what is the physical output of the assay? Despite the different languages of the disciplines, having a shared understanding of the goals of the research, the experimental design, the techniques to be used and their limitations early on is very important for a long and fruitful collaboration.

When I first started designing nanoparticles for delivering siRNA to endothelial cells, one of the things I had to do was to identify a lead candidate, and then optimize it. Along with other scientists in the lab, we spent a significant amount of time ensuring that the nanoparticle size and function were consistent across many and open communication to happen, and the inevitable conversations about different techniques to use ensured our experiments were optimized. Throughout the experience, I found that the most critical component to any need to be best friends with your collaborators (although it is fun when that happens), but you do need to know that you are playing on the same team.

Given that molecular biologists, geneticists and physicians are uncovering relationships between three-dimensional DNA structure, gene function and molecular phenotype, and given the fact that many of these interactions take place on a nanometre scale, the demand for nanotechnological tools to study biology will continue to grow. There is no doubt that improved communication between these once disparate fields will be critical if we are to efficiently solve the myriad of exciting scientific problems at their interface.

JAMES E. DAHLMAN just completed his $\mathrm{PhD}$ at the Harvard-MIT Division of Health Sciences and Technology, Massachusetts Institute of Technology, Cambridge, Massachusetts 02142, USA, and is now at the Broad Institute of MIT and Harvard, Cambridge, Massachusetts 02142, USA. e-mail: jdahlman@mit.edu collaboration is trust: you do not necessarily 